\title{
CORE)-(OES
}

CIÊNCIA E TECNOLOGIA

\section{ANÁLISE COMPARATIVA ENTRE UM SISTEMA SOLAR COM RASTREIO E UM FIXO}

\author{
DANIEL SiLVA LiMA \\ Universidade Federal Rural do Semi-Árido (UFERSA) \\ $<$ daniel_silva_lima@outlook.com> \\ DOI: <https://doi.org/10.21439/conexoes.v14i4.1334>
}

\begin{abstract}
Resumo. A energia solar é uma fonte alternativa às fontes tradicionais que vem ganhando espaço ano após ano. A partir da conversão da radiação solar incidente em um módulo fotovoltaico é possível obter energia elétrica. No entanto a baixa eficiência na conversão ainda é um ponto negativo para consolidação da tecnologia solar fotovoltaica. O presente trabalho teve como objetivo construir um sistema fotovoltaico com seguimento solar em um eixo e compará-lo a um sistema estático. Foram utilizados sensores, resistores, dispositivos elétricos, equação para descrever a trajetória do sol no caso do sistema com seguidor solar e um Arduino $\mathbb{R}$ Mega para armazenamento, sensoriamento, controle e leitura dos dados das cinco horas da manhã as seis horas da noite. Os resultados do sistema com rastreio se sobressaíram em relação ao sistema estático, onde foi constatado um ganho de até $23 \%$ na geração de energia. Diante disso, foi possível observar que o sistema com rastreio solar é uma opção a ser considerada em sistemas fotovoltaicos.
\end{abstract}

Palavras-chaves: Energia Solar. Eficiência. Rastreio Solar.

\section{COMPARATIVE ANALYSIS BETWEEN A SOLAR SYSTEM WITH TRACKING AND A FIXED ONE}

\begin{abstract}
Solar energy is an alternative source to traditional sources who have been growing annually. From the conversion of incident solar radiation into a photovoltaic module it is possible to obtain electric energy. However the low conversion efficiency is still a negative point for consolidation of solar photovoltaic technology. The objective of this work was to construct a photovoltaic system with solar tracking on an axis and compare it to a static system. We used sensors, resistors, electrical devices, equation to

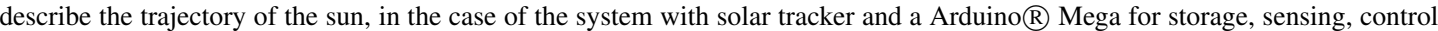
and reading data from five o'clock in the morning to six o'clock at night. The results of the system with tracing have stood out in relation to the static system, where a gain of up to $23 \%$ in the generation of energy was found. Given this, it was possible to observe the system with solar screening and an option to be considerable in photovoltaic systems.
\end{abstract}

Keywords: Solar Energy. Efficiency. Solar Tracking.

\section{INTRODUÇÃO}

As fontes alternativas de energia vêm ganhando espaço no cenário mundial devido a fatores como: Crescimento da população mundial levando ao aumento do consumo energético global de tal forma que as fontes convencionais sozinhas não estão respondendo à demanda atual; aumento constante da emissão de gases poluentes na atmosfera devido ao uso de fontes não renováveis de energia como o petróleo e o carvão mineral; natureza finita dos combustíveis fósseis.

Entre as fontes que surgem como alternativas está a solar que além de ser aproveitada de forma direta é de grande importância para as outras fontes de energia, atuando de forma indireta ou passiva no aproveitamento da energia eólica e dos oceanos, que são ocasi- onadas pela diferença de temperatura entre regiões diversas da terra. Biomassa e a energia hidráulica que se originam de processos de fotossíntese e diferença de potencial dependem da influência da radiação solar são outros exemplos da importância do Sol na matriz energética mundial. Umas das formas de aproveitamento da energia solar é dada a partir da captação e posterior conversão das radiações eletromagnéticas incidentes no planeta em energia elétrica são os módulos fotovoltai$\cos$.

Devido à baixa eficiência dos sistemas fotovoltaicos são necessários estudos para a maximização da sua conversão, tornando tal fonte mais eficiente. Estudos vêm sendo realizados com rastreadores solares adicionados a painéis fotovoltaicos, onde eles conseguem rastrear o 
sol, aumentando a geração de energia elétrica dos sistemas fotovoltaicos. Logo, o presente trabalho analisa de forma experimental a eficiência na produção de energia elétrica em dois sistemas fotovoltaicos onde um é fixo e o outro móvel, com mobilidade em um eixo controlado por um rastreador solar.

\section{POSIÇÃO DO SOL}

Para implementação de um sistema de rastreio do sol é necessário inicialmente um estudo sobre a posição do Sol ao longo do dia e do ano naquele local. A posição para cada instante de tempo pode ser determinada a partir dos ângulos formados entre os raios solares incidentes, o módulo fotovoltaico e os eixos cardeais Norte-Sul e Leste-Oeste (VIEIRA et al., 2014). Os ângulos estão ilustrados na Figura 1

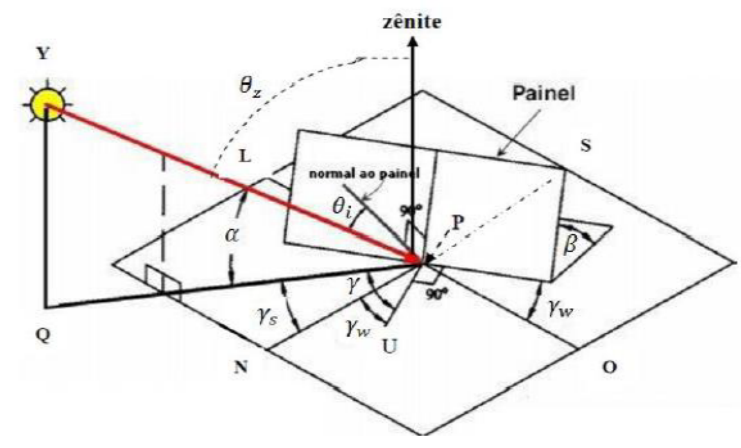

Figura 1: Ângulos formados entre os raios incidentes, eixos cardeais e módulo fotovoltaico.

Fonte: Ribeiro, Prado e Gonçalves (2012).

A posição do sol pode ser descrita a partir dos ângulos de azimute $\gamma_{w}$ e altitude solar $\alpha$. Relacionando tais ângulos visualizados na Figura 1 junto ao ângulo de declinação solar $\delta$ é descrita a equação que descreve o ângulo de ajuste do módulo fotovoltaico que maximiza a conversão dos raios solares incidentes em energia elétrica. Segundo (MASTERS, 2004), o ângulo $\alpha$ é dado pela Equação 1 onde $L$ representa a latitude do local.

$$
\alpha=90^{\circ}-L+\gamma
$$

A partir do ângulo de altitude solar é possível determinar a inclinação $\beta$ que melhor adequa-se ao módulo fotovoltaico para maximização da conversão. A Equação 2 descreve o ângulo de inclinação $\beta$ visualizado na Figura 1

$$
\beta=90^{\circ}-\alpha
$$

A identificação do ângulo de inclinação para diferente posições do sol no decorrer do dia possibilita a utilização de um seguidor solar para que os raios solares incidam no módulo perpendicularmente otimizando o sistema fotovoltaico.

\section{SEGUIDORES SOLARES}

Um sistema de seguimento solar pode ser utilizado em basicamente todas as aplicações que usem energia solar, ou seja, tanto em painéis fotovoltaicos como em coletores planos e concentradores solares usados no aquecimento de água. Existem casos em que um sistema de seguimento solar, apesar de incrementar em cerca de $20 \%$ o preço total de um sistema de geração fotovoltaica de energia, pode vir a aumentar em algo em torno de $40 \%$ as receitas geradas. Os seguidores fotovoltaicos também apresentam geralmente um baixo custo de manutenção (GIL et al., 2009).

Considerando as atenuações da atmosfera, o uso de rastreador de sol leste -oeste apresenta a vantagem teórica de aumentar em até $48 \%$ a capacidade de aproveitamento dos raios solares para conversão em energia em dias sem nuvens. A tecnologia fotovoltaica se destaca pelo fato de possuir grande robustez associada aos painéis fotovoltaicos e sua reduzida manutenção (SHAYANI, 2006). Para a montagem de um sistema com rastreio é necessário inicialmente um estudo da área de implantação da tecnologia junto a um sistema de controle bem estruturado.

Existem formas diferentes de se obter o controle de seguimento. $\mathrm{O}$ controle pode ser realizado em malha aberta, ou seja, através de algoritmos que efetuam o cálculo da posição do sol, em malha fechada, com o uso de sensores ou ainda de forma mista, como o nome já diz, efetua a interação entre sensores e o algoritmo (CORTEZ, 2012). Em uma configuração mais completa, um sistema de seguimento pode ser composto por:

(i) Aquisição: Permite a inicialização da orientação inicial do módulo fotovoltaico para dias do ano através do fornecimento dos ângulos solares necessários para o rastreio do sol;

(ii) Sensores: É o responsável pela procura do sol no decorrer do dia, sem a necessidade dos ângulos solares em conjunto com a hora solar. Entre os com ponentes estão os fototransistores, fotosensores, fotodiodo entre outros;

(iii) Controlador: Circuito elétrico podendo ou não estar em conjunto com microcontroladores, onde os sinais emitidos dos sensores são processados de acordo com a lógica de controle ou programação e de onde saem os sinais de comando para os atuadores; 
(iv) Acionador: Realiza a movimentação do sistema a partir dos sinais vindos do controlador, sendo geralmente utilizados motores de corrente contínua devido ao fácil acesso.

A Figura 2 ilustra a composição de um sistema de rastreio solar a partir de um diagrama.

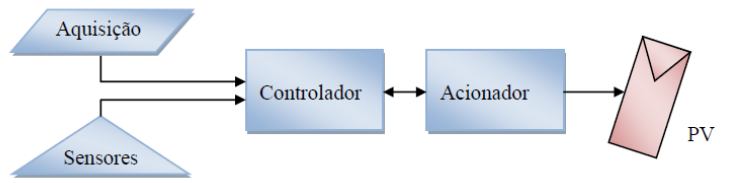

Figura 2: Esboço de um diagrama em blocos do sistema de rastreio solar.

Fonte: Ribeiro, Prado e Gonçalves (2012).

\section{MATERIAIS E MÉTODOS}

O presente trabalho visa inicialmente a elaboração de uma equação junto a um sistema de seguimento solar que efetuará o acionamento de um motor de passo, este último está acoplado a uma estrutura com um grau de liberdade. O intuito é realizar o acompanhamento da posição do sol durante o dia para uma localidade específica no caso trata-se da cidade de Mossoró-RN. É realizado também a comparação entre um sistema estático e outro móvel para observar a influência de um seguidor solar quanto a geração de energia de um sistema fotovoltaico.

Os materiais necessários para realização do experimento foram:

(a) Resistores variados para dissipar potência gerada pelos painéis e para atuar como divisores de tensão

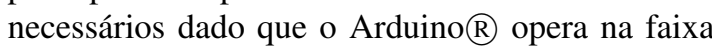
entre $0 \mathrm{~V}$ a $5 \mathrm{~V}$;

(b) Dois módulos fotovoltaicos da Suntech modelo STP020S-12Cb;

(c) Um suporte para fixação do sistema móvel;

(d) Um motor de passo de corrente contínua para movimentação do painel fotovoltaico;

(e) Três sensores de corrente modelo ACS712ELC-30 para medição das correntes consumidas pelos resistores e consumida pelo motor de passo;

(f) Um sensor de temperatura e umidade modelo DHT11;

(g) Sensores LDR's para medir radiação incidente; (h) Um Arduino $囚$ Mega;

(i) Um SD shield 2.0 junto a um cartão de memória para armazenamento dos dados;

(j) Além de bateria para alimentação do motor de passo e fiação em geral.

A estrutura e o motor de passo junto ao seu drive foram reaproveitados de experimentos anteriores. E alguns itens como sensores de corrente, fios de conexão e resistências foram requeridos através de recursos financeiros próprios. Todos os outros materiais foram obtidos sem custos.

O posicionamento do módulo fotovoltaico do sistema móvel foi realizado via uma equação que descreve a trajetória do sol em relação à hora do dia. Para gerar tal equação inicialmente foi observado a relação entre posição do sol e hora do dia empiricamente e posteriormente foi usado o software Sun Earth Tools, que gera o ângulo de altura solar para várias horas do dia, neste caso foram obtidos os ângulos de altura solar, azimute entre outros de 15 em 15 minutos a partir das 6:00h da manhã até pouco mais das 18:00h da noite.

Através das Equações 1] e 2 descritas anteriormente foi possível determinar a inclinação do painel para determinada hora do dia. A partir disso foi aplicado o modelo de regressão polinomial de grau dois para traçar uma curva que relacione o ângulo de altura solar e a hora do dia. A escolha por tal modelo de regressão se deve ao fato de observar através dos dados obtidos que a relação entre a posição do sol através do ângulo de elevação descrevia uma trajetória parabólica. A Figura 3 mostra o modelo matemático escolhido junto a função que descreve tal relação entre a variável dependente que no caso é o ângulo de altura solar e a variável independente que neste caso é a hora do dia.

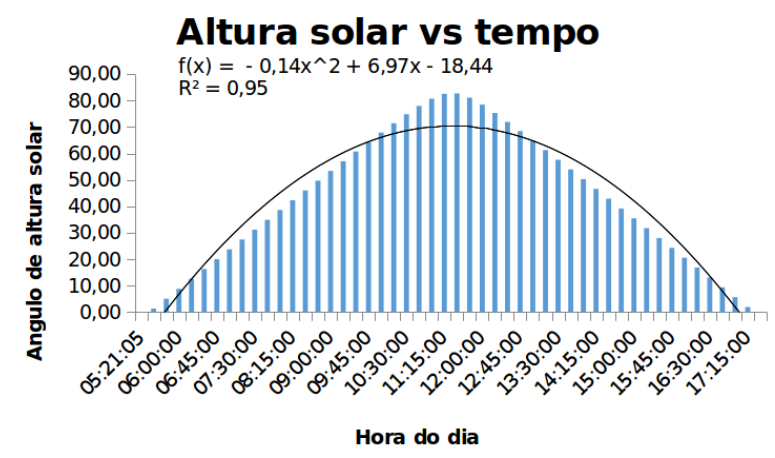

Figura 3: Relação do ângulo de altura solar com a hora do dia

Observa-se um coeficiente de determinação de $94,6 \%$, ou seja, quase $95 \%$ da variável dependente, 
neste caso, da altura solar podem ser explicados pelos regressores presentes na equação 3 .

$$
\alpha=-0,136 t^{2}+6,966 t-18,44^{\circ}
$$

Relacionando as Equações 2 e 3 é obtido a Equação 4 descrita a seguir.

$$
\beta=90^{\circ}-0,136 t^{2}+6,966 t-18,44^{\circ}
$$

Logo, o ângulo de inclinação do painel móvel irá descrever uma trajetória parabólica com o intuito de que os raios solares atinjam a superfície do painel perpendicularmente. Então, é necessário relacionar o ângulo de inclinação do módulo fotovoltaico com a quantidade de passos que o motor irá executar via o controle do Arduinoß. Sabendo que um passo do motor HT23-397 equivale a $1,8^{\circ}$ e que a estrutura permite que o painel tenha uma variação angular de $90^{\circ}$ então através de uma divisão temos que o número de passos executados durante o dia para que ocorra o rastreamento do sol é igual a 50. A variação do ângulo de inclinação do módulo do sistema móvel ocorrerá de 15 em 15 minutos onde o primeiro passo do motor era realizado as 8:00h da manhã enquanto o último passo era realizado as 17:45h.

O sistema com o módulo fotovoltaico fixo foi inserido diretamente ao chão a uma inclinação de aproximadamente 15 graus. Com isso, após a fase de estudos e aquisição dos materiais e com todo o sistema de automação desenvolvido foram realizados os testes entre os dias 19 a 21 de outubro de 2016 na cidade de Mossoró$\mathrm{RN}$, onde ambos os sistemas foram implantados no local já caracterizado na noite do dia 18 de outubro de 2016. A prática foi finalizada no dia 21 de outubro e por volta das 18 horas o sistema foi retirado de seu funcionamento para posterior análise dos dados coletados. Na Figura 4 é apresentado o sistema móvel.

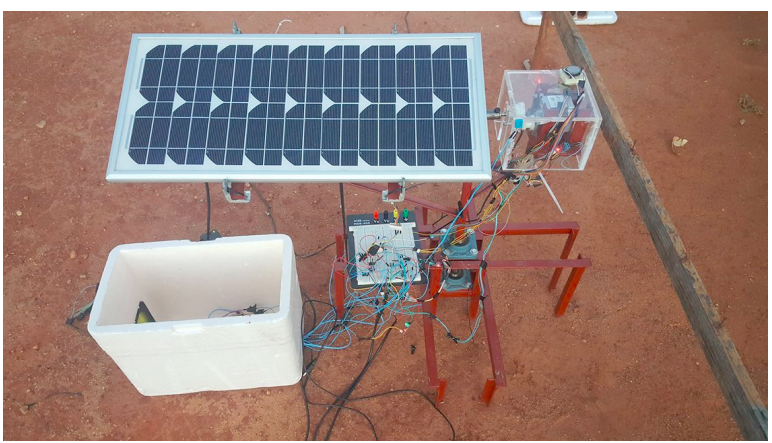

Figura 4: Sistema fotovoltaixo móvel.

Em ambos os módulos foram adicionados resistores apropriados de aproximadamente $20 \Omega$ para dissipar a potência gerada pelo painel durante o seu funcionamento e foi adicionado um cooler no recipiente em que

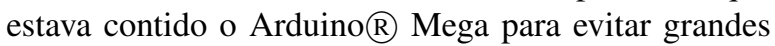
aumentos de temperatura e futuras perdas de dados. Os testes foram realizados ao mesmo tempo para ambos os sistemas em um período de 3 dias. O desempenho foi monitorado 24 horas durante os 3 dias, no entanto os dados fornecidos pelos sensores foram analisados entre as 05:00h e 17:30h, tal intervalo foi determinado pois foi perceptível que fora desse intervalo a geração de energia era desprezível.

\section{RESULTADOS E DISCUSSÕES}

A partir do levantamento e armazenamento dos dados de tensão e corrente de ambos os sistemas foi possível estimar a potência e energia gerada por ambos os sistemas. Na Tabela 1 é apresentado o desempenho dos dois sistemas assim como o ganho do sistema móvel quando comparado ao sistema. Além do desempenho e do ganho é verificado as condições climáticas em cada dia do experimento.

Observa-se na Tabela 1 que nos três dias de ensaio, o sistema móvel gerou mais energia do que o sistema fixo, onde o ganho máximo ficou em torno de $23 \%$. Já o ganho médio foi de aproximadamente $16 \%$. Ainda na Tabela 1 é possível perceber que no dia nublado o desempenho do sistema móvel perante o sistema fixo foi maior do que nos outros dois dias. Vale ressaltar que a forma de rastreio via equação do tempo não sofre interferência da nebulosidade, pois o seguimento do sol é realizado em relação a sua posição com o tempo e não em relação à luminosidade. Esta é uma das vantagens deste tipo de rastreio.

Mesmo com um ganho próximo a $23 \%$, é observado que a energia gerada diminui com a nebulosidade, isso ocorre devido à geração de radiação difusa que não é aproveitada na conversão para energia elétrica. As Figuras 5, 6, e 7 mostram graficamente a comparação da quantidade de energia gerada pelos sistemas.

As Figuras 5, 6 e 7 mostram que a energia gerada pelo sistema móvel esteve na maioria das vezes superior à energia gerada pelo sistema estático, onde essa vantagem era bem perceptível no começo da manhã quando a radiação já existia de tal forma que o painel móvel estava bem posicionado para captação dos raios solares incidentes assim como após as 15:00 horas quando o sol começa a se pôr em Mossoró-RN para tal época do ano.

A diminuição da radiação incidente junto a variação do ângulo de incidência dos raios solares após as 14:00 horas acarretava um decréscimo de energia gerada. É observado que o rendimento superior do sistema móvel 


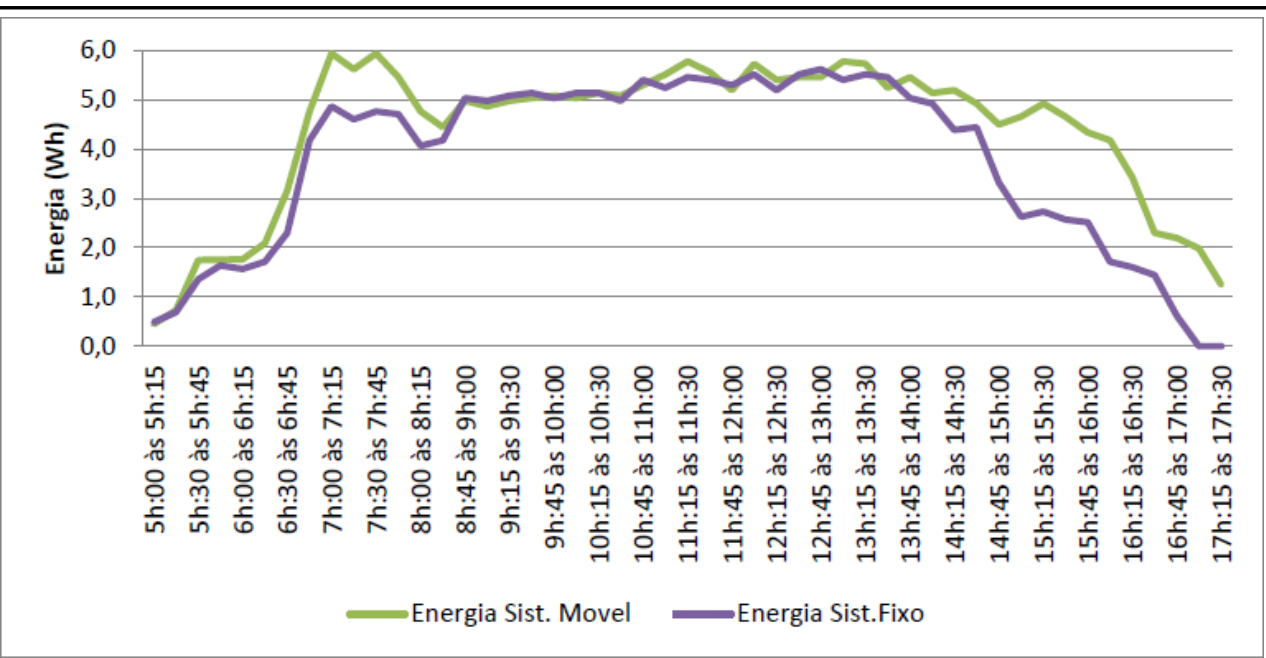

Figura 5: Comparação do desempenho dos sistemas móvel e estático no dia 1.

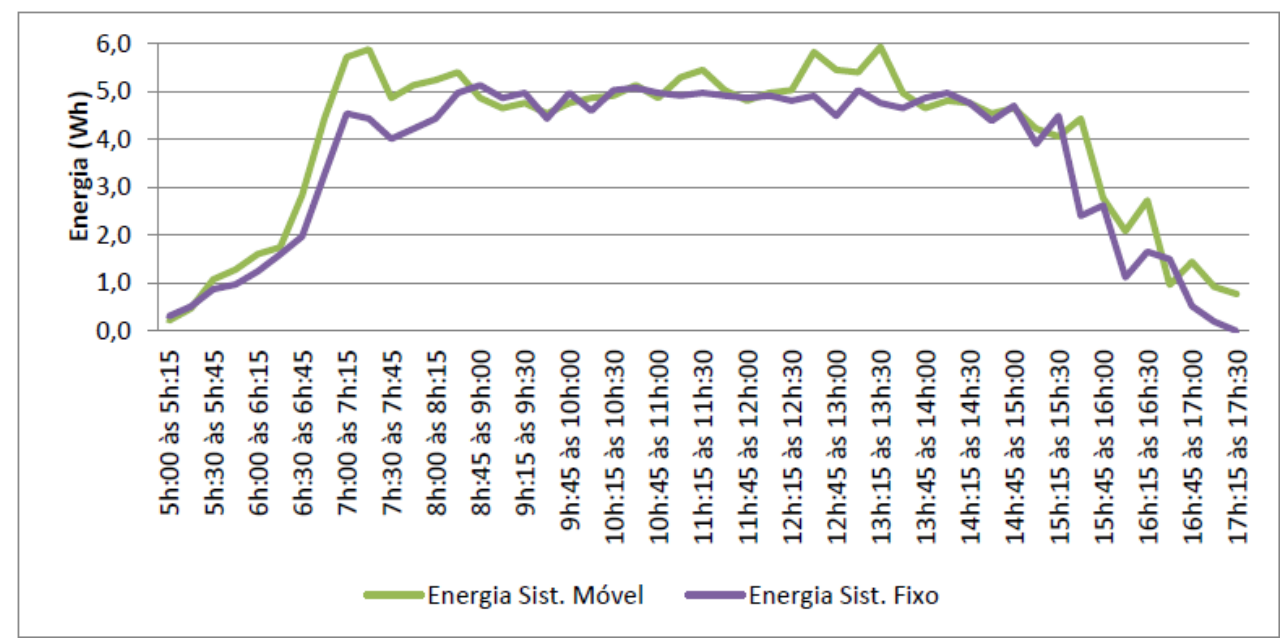

Figura 6: Comparação do desempenho dos sistemas móvel e estático no dia 2.

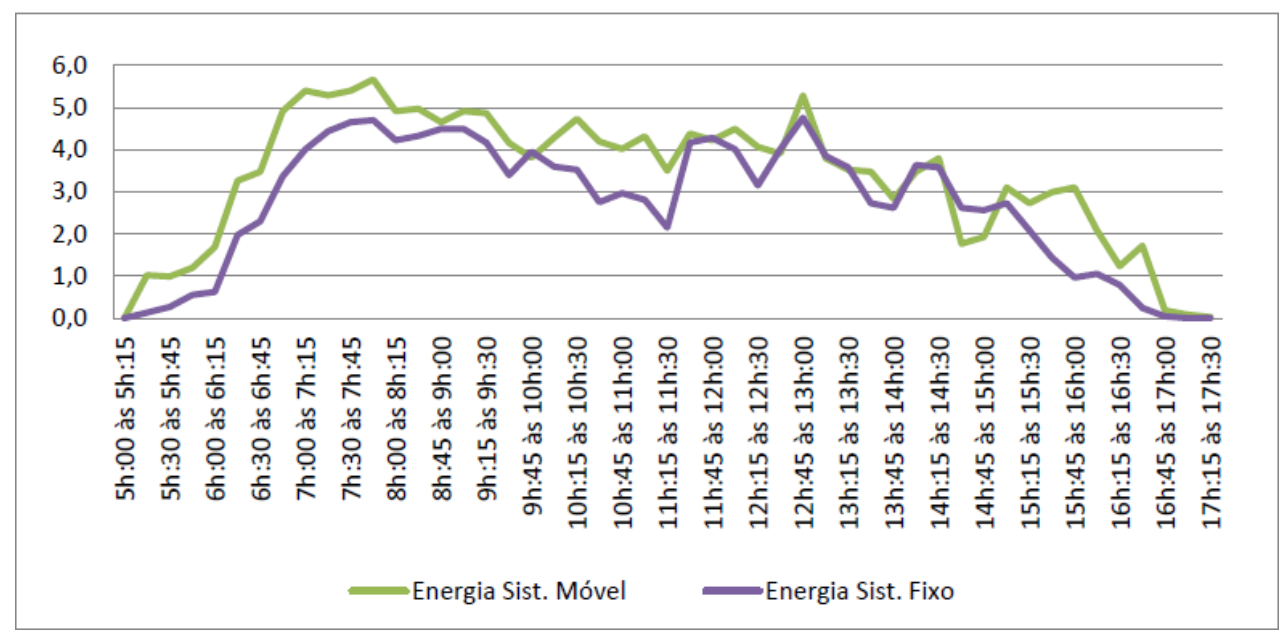

Figura 7: Comparação do desempenho dos sistemas móvel e estático no dia 3.

Conex. Ci. e Tecnol. Fortaleza/CE, v. 14, n. 4, p. 146 - 151, ago. 2020

Artigo submetido em 22 jul. 2017 e aceito em 31 jan. 2020. 


\begin{tabular}{ccccc}
\hline \multirow{2}{*}{ Dia } & $\begin{array}{c}\text { Energia Sist. Móvel } \\
(\mathbf{W h})\end{array}$ & $\begin{array}{c}\text { Energia Sist. Estát. } \\
(\mathbf{W h})\end{array}$ & $\begin{array}{c}\text { Ganho } \\
(\mathbf{\%})\end{array}$ & $\begin{array}{c}\text { Condições } \\
\text { meteorológicas }\end{array}$ \\
\hline 1 & 214,25 & 184,33 & 16,23 & Nuvens esparsas \\
2 & 195,29 & 176,71 & 10,51 & Nuvens esparsas \\
3 & 163,83 & 132,74 & 23,42 & Parcialmente Nublado \\
\hline Média & $\mathbf{1 9 1 , 1 2}$ & $\mathbf{1 6 4 , 5 9}$ & $\mathbf{1 6 , 1 2}$ & - \\
\hline
\end{tabular}

em relação ao fixo ocorreu, mas sem grandes diferenças, devido a fatos como o sistema ser composto apenas por um módulo fotovoltaico e as condições de alta incidência dos raios solares existentes em Mossoró-RN, logo o rendimento do móvel quando comparado ao fixo é de baixo ganho.

Vale ressaltar que em nenhum momento ocorreu à desorientação do painel, o mesmo sempre esteve seguindo a equação 4 descrita anteriormente, tal fato pode ser observando quando comparamos o desempenho dos sistemas fixo e móvel no dia parcialmente nublado, nesse dia ocorreu o maior ganho do sistema com rastreio em relação ao fixo. Caso o rastreio fosse realizado com sensores $L D R$ 's poderia ocorrer justamente a desorientação do painel móvel diminuindo assim a incidência dos raios solares no painel fotovoltaico.

\section{CONCLUSÕES}

O sistema fotovoltaico com rastreio solar mostra-se uma alternativa de baixo custo para aumentar a eficiência dos geradores fotovoltaicos, ou seja, eleva a energia produzida pelo sistema. A pesquisa comparou dois sistemas, um fixo e outro acoplado ao seguidor solar, com o objetivo de observar experimentalmente a melhora do sistema ao seguir o sol durante o dia.

Foi possível concluir que o ganho médio de aproximadamente $16 \%$ do sistema com mobilidade em um eixo em relação ao fixo é satisfatório quando comparado a outros trabalhos inseridos nas mesmas condições climáticas presentes na literatura. Ambos os sistemas mostram uma relação inversamente proporcional entre a umidade relativa do ar e a energia gerada e uma relação diretamente proporcional entre a temperatura e a energia gerada pelos painéis fixo e móvel durante os dias de experimento. A potência gerada nos dias 19 e 20 de outubro eram sempre superiores a potência nominal do painel, fato ocorrido entre outros motivos devido à radiação elevada a qual o trabalho estava inserido interferindo diretamente na corrente gerada pelo módulo fotovoltaico.

Diferenças significativas na potência gerada pelos sistemas eram visualizadas no início da manhã e após as
15 horas. No dia parcialmente nublado o sistema móvel apresentou ganho médio de quase $23,4 \%$ em relação ao sistema fixo, neste dia ocorreu a maior diferença de rendimento entre os sistemas móvel e fixo.

\section{REFERÊNCIAS}

CORTEZ, R. J. M. Sistema de Seguimento Solar em Produção de Energia Fotovoltaica. Dissertação (Mestrado Integrado em Engenharia Eletrotécnica e de Computadores) - Faculdade de Engenharia do Porto - FEUP, Portugal, 2012.

MASTERS, G. M. Renewable and efficient electric power systems. 1. ed. New Jersey: John Wiley \& Sons, 2004.

RIBEIRO, S. C.; PRADO, P. P. L. d.; GONçALVES, J. B. Projeto e desenvolvimento de um rastreador solar para painéis fotovoltaicos. In: ASSOCIAçãO EDUCACIONAL DOM BOSCO. SIMPÓSIO DE EXCELêNCIA EM GESTÃO E TECNOLOGIA. Rio de Janeiro, 2012. v. 1, n. 11, p. 1-10.

SHAYANI, R. A. Medição do rendimento global de um sistema fotovoltaico isolado utilizando módulos de 32 células. Dissertação (Mestrado em Engenharia Elétrica)-Departamento de Engenharia) Universidade de Brasília, Brasília, 2006. 205 f.

VIEIRA, R. G. et al. Análise comparativa do desempenho entre um painel solar estático e com rastreamento no município de Mossoró-RN. Dissertação (Pos-Graduacao em Sistemas de Comunicacao e Automacao) - Universidade Federal Rural do Semi-Árido, Mossoró - RN, 2014. 